\title{
AN ASSESSMENT OF THE RELATIONSHIPS BETWEEN BALL FLIGHT RESULTS, IMPACT FACTORS, AND GOLF PERFORMANCE
}

original paper

(c) Wroclaw University of Health and Sport Sciences

DOI: https://doi.org/10.5114/hm.2021.104180

MATĚJ BROŽKA, TOMÁŠ GRYC, PETR MIŘÁTSKÝ, FRATIŠEK ZAHÁLKA

Sport Research Center, Faculty of Physical Education and Sport, Charles University, Prague, Czech Republic

\begin{abstract}
Purpose. The aim of the present study was to investigate the relationship between golf players' performance, impact factors, and ball flight results with individual clubs when performing a full swing.

Methods. The study involved 9 amateur golfers who performed a full swing test with 4 different clubs (short, middle, long iron, and driver) and then played a tournament round of golf. The players' performance was assessed by handicap and by game statistics from the tournament. The ball flight results were based on the resulting carry distance and the resulting side deviation from the target line. The impact factors were the initial ball speed, the club head speed, the smash factor, the face angle, the club path, and the face to path. Relationships between the players' performance, impact factors, and ball flight results were investigated.

Results. A significant relationship was found between the resulting ball flight distance and handicap for long iron $(r=-0.85$; $p<0.01)$ and driver $(r=-0.9 ; p<0.01)$. Conversely, the resulting ball flight side deviation from the target line correlated with handicap in short iron $(r=0.78 ; p=0.02)$.

Conclusions. Ball flight distance of long iron and driver and ball flight side deviation of short iron are key attributes of performance. The results suggest that in order to improve long term performance, players should aim for maximal distance with drivers and long irons (high smash factor) and for maximal accuracy with short irons (low face angle).
\end{abstract}

Key words: diagnostics, analysis, golf, full swing, TrackMan

\section{Introduction}

Driving, iron strokes, approach strokes, chips around the green, and putting are golf skills that combined determine the outcome performance in golf. In general, the player's goal is to play every stroke in the desired direction with correct distance. The ball flight results are influenced by the club head kinematics at impact, so-called impact factors [1-3]. Impact factors directly influence the resulting direction (both initial start direction and any curvature) and distance of the ball flight, which subsequently influence a players' performance. The resulting ball flight distance is directly dependent on initial ball speed after contact, club head speed prior to contact, and the quality of the club-ball interaction - often referred to as the smash factor [1, $4,5]$. The resulting ball flight distance is the main determinant of the success for winning tournaments and the amount of earnings in Professional Golfers' Association (PGA) golfers [6]. Additionally, the club head speed strongly correlates with the players' performance (handicap) [7, 8] and in many cases is used as the sole indicator of performance [9-15]. Furthermore, the resulting distance of the ball flight is dependent on launch angle (vertical angle the ball takes off relative to the horizon) and spin rate (the amount of spin on the golf ball immediately after impact). The resulting direction is measured by side deviation from the target line, which is determined by the ball flight trajectory (curve) and the launch direction (initial direction the ball starts relative to the target line). The ball flight trajectory in the horizontal plane is most affected by the spin axis (the tilt angle relative to the horizon of the golf ball's resulting rotational axis immediately after separation from the club face), which is determined by the angle difference between the face angle (the direc-

Correspondence address: Matěj Brožka, Sport Research Center, Faculty of Physical Education and Sport, Charles University, José Martího 269/31, 162 52, Prague 6, Czech Republic, e-mail: brozka.matej@hotmail.com

Received: November 25, 2019

Accepted for publication: September 18, 2020

Citation: Brožka M, Gryc T, Miřátský P, Zahálka F. An assessment of the relationships between ball flight results, impact factors, and golf performance. Hum Mov. 2022;23(1):1-9; doi: https://oi.org/10.5114/hm.2021.104180. 
tion the club face aim relative to the target line at impact) and the club path (the direction the club head moves relative to the target line at impact). Launch direction is affected in $70-85 \%$ by face angle and in $15-30 \%$ by club path, depending on the club type $[5,16]$. With a full swing, smaller errors in side deviation are essential to improve finishing proximity from the hole [17]. The face angle at impact was the most important factor in the launch direction (explaining $82 \%$ of the variance) in a study by Miura [18].

The ball flight results and the impact factors influence amateur players' handicap level, where a lower handicap indicates better performance [1, 19-22]. Professional players are evaluated by the amount of earnings [6, 23-25]. However, performance in both categories can be assessed by the average number of strokes or number of strokes for a single round [26]. Additionally, players' performance can be determined by game statistics, such as the percentage of fairways in regulation (percentage of drives finishing on the fairway), the percentage of greens in regulation (percentage of greens hit in the recommended number of strokes - the first stroke on par 3s, the second stroke on par $4 \mathrm{~s}$, and the third stroke on par 5s), putts per round, etc. Furthermore, game statistics significantly correlate with the average number of strokes and earnings on PGA Tour, where the percentage of greens in regulation has the strongest correlation from all game statistics [27, 28].

Previous studies have quantified the relationship between club head kinematics and early ball flight characteristics when using drivers [4], as well as the relationship between the variability of impact factors and players' performance. To the best of our knowledge, there is no complex study examining the relationship between impact factors, players' performance, and game statistics. Furthermore, no studies have inves- tigated these relationships with a number of individual clubs. Previous research has only considered the relationship between players' performance and the variability of impact factors, not with the magnitude of impact factors. Including game statistics as indicators of players' performance and its relationship with impact factors and ball flight results could be beneficial for coaches. Finding the key parameters in the impact factors and ball flight results should be helpful in improving players' performance. Consequently, the purpose of this study was to investigate the relationship between players' performance, impact factors, and ball flight results with individual clubs when performing a full swing.

\section{Material and methods}

\section{Participants and design}

The research sample consisted of amateur male golf players ( $n=9$; age: $36.2 \pm 7.9$ years; height: $182.1 \pm$ $6.0 \mathrm{~cm}$; mass: $85.5 \pm 13.2 \mathrm{~kg}$; handicap: $7.2 \pm 3.5$; golf experience: $11.2 \pm 4.5$ years) who volunteered to participate in the study. The individuals were intentionally selected from among amateur golf players who participated in a golf tournament.

The study followed the ethical standards in sport and exercise science research [29]. The experiment was held before an 18-hole tournament on the driving range as players warmed-up. The participants were familiarized with the full swing test and took 20 total strokes, 5 strokes with each of the following clubs in this order: short iron (pitching wedge), middle iron (eight iron), long iron (5 iron), and driver. The ball flight results from the full swing test were evaluated by the resulting ball flight distance and resulting ball flight side deviation from the target line. Target line was set

Table 1. Ball flight results and selected impact factors used in the study

\begin{tabular}{ll}
\hline Parameters & \multicolumn{1}{c}{ Definitions } \\
\hline $\begin{array}{l}\text { Resulting ball flight distance } \\
\begin{array}{l}\text { Resulting ball flight side deviation } \\
\text { from the target line }\end{array}\end{array}$ & $\begin{array}{l}\text { Distance between starting position and final carry impact position of the ball } \\
\text { Distance between target line and final carry impact position of the ball. } \\
\text { Distance line is perpendicular to target line }\end{array}$ \\
$\begin{array}{l}\text { Club head speed } \\
\text { The speed of the golf ball immediately after impact } \\
\text { Thash factor }\end{array}$ & $\begin{array}{l}\text { The speed of the club head as travelling immediately prior to impact } \\
\text { Face angle }\end{array}$ \\
Club path & The direction the club face pointed (right or left) at impact \\
Face to path & The direction the club head moving (right or left) at impact \\
\hline
\end{tabular}


to the mark in the middle of the driving range for all clubs. The selected impact factors were the initial ball speed, the club head speed, the smash factor, the face angle, the club path, and the face to path. The resulting ball flight distance and resulting ball flight side deviation from the target line, along with impact factors, were recorded by using the 3D Doppler Radar TrackMan 4 (TrackMan, Denmark), which enables 3D real-time tracking of the club head and the ball and is commonly used by elite golfers at the world's highest professional tournament series, the PGA Tour. The validity of the device was verified by Leach et al. [30]. Table 1 defines the selected impact factors used in the study. The players' performance was assessed by handicap, which indicates long-term performance, and by game statistics from the tournament as actual performance on the day of the test. The game statistics evaluated in the tournament were the number of strokes, the percentage of greens in regulation, and the percentage of fairways in regulation.

\section{Mathematical-statistical processing and statistical analysis}

The normal distribution of the dataset was verified by the Shapiro-Wilk test for each parameter. The results were first processed with mathematical and statistical methods by using Microsoft Excel (Redmond, USA) to determine the central tendency (mean, standard deviation). All parameters were reported in absolute values. Significant differences in the impact factors and in ball flight results between different clubs were evaluated by one-way ANOVA with Tukey's post-hoc test. To examine the relationship between the players' performance, the impact factors, and the ball flight results, Pearson's product-moment correlation coefficient $(r)$ was applied in the R v. 3.5.2 software (Vienna, Austria). The value of the relationship between parameters was judged as recommended by Portney and Watkins [31], i.e. 0.00-0.25: small or no relationship, 0.26-0.50: low relationship, 0.51-0.75: medium or good relationship, and 0.76-1.0: good to excellent relationship. The level of significance for all analyses was set at $\alpha=$ 0.05 to reject the zero hypothesis.

\section{Ethical approval}

The research related to human use has complied with all the relevant national regulations and institutional policies, has followed the tenets of the Declaration of Helsinki, and has been approved by the Ethics Committee of the Faculty of Physical Education and Sport, Charles University, under the number EC 179/2019.

\section{Informed consent}

Informed consent has been obtained from all individuals included in this study.

\section{Results}

The players achieved a number of strokes of $77.1 \pm$ 3.4 , a percentage of greens in regulation of $54.9 \pm 10.3 \%$, and a percentage of fairways in regulation of $44.4 \pm$ $15.3 \%$. A significant relationship between handicap and the percentage of greens in regulation $(r=-0.66$; $p=0.05$ ) was found. No significant relationships were observed between: handicap and the percentage of fairways in regulation; handicap and the number of strokes; the number of strokes and the percentage of greens in regulation; the number of strokes and the percentage of fairways in regulation; the percentage of fairways in regulation and the percentage of greens in regulation.

Table 2 shows the absolute means and standard deviations of the ball flight results and the impact factors for each club. Significant differences were found in the resulting ball flight distance and in the ball speed between particular clubs $(p<0.05)$. There were also significant differences in the club head speed between the clubs $(p<0.05)$, except for the comparison between short iron and middle iron $(p=0.2)$. As the length of the club increased, the club head speed, the initial ball speed, and the resulting ball flight distance increased. Significant differences were observed in the smash factor between particular clubs $(p<0.05)$, with an exception of the comparison between middle iron and long iron $(p=0.3)$. A significant difference was revealed in the resulting ball flight side deviation between short iron and driver $(p<0.01)$, between middle iron and driver $(p<0.01)$, and between long iron and driver $(p=0.01)$. No significant difference in the club path, the face angle, or the face to path between the clubs $(p>0.05)$ was demonstrated.

Table 3A shows the relationships between the ball flight results and impact factors: the club head speed, the initial ball speed, and the smash factor. A significant relationship was found between the resulting ball flight distance and the initial ball speed for all clubs (short iron: $r=0.8, p=0.01$; middle iron: $r=$ $0.82, p=0.01$; long iron: $r=0.86, p<0.01$; driver: $r=0.87, p<0.01)$ and between the resulting ball flight distance and the smash factor for all clubs (short iron: $r=0.67, p=0.05$; middle iron: $r=0.81, p=0.01$; long iron: $r=0.7, p=0.04$; driver: $r=0.76, p=0.02$ ). Table 3B presents the relationships between the ball flight results and impact factors: the club path, the 


\section{HUMAN MOVEMENT}

M. Brožka T. Gryc, P. Miřátský, F. Zahálka, Stroke performance in golf

Table 2. Mean $(\varnothing)$ and standard deviation $(S D)$ of the ball flight results and the impact factors for each club

\begin{tabular}{|c|c|c|c|c|c|c|c|c|c|}
\hline \multirow{2}{*}{ Club } & & \multicolumn{6}{|c|}{ Impact factors } & \multicolumn{2}{|c|}{ Ball flight results } \\
\hline & & $\mathrm{CHS}(\mathrm{m} / \mathrm{s})$ & $\operatorname{IBS}(\mathrm{m} / \mathrm{s})$ & $\mathrm{SF}$ & $\mathrm{CP}\left({ }^{\circ}\right)$ & $\mathrm{FA}\left({ }^{\circ}\right)$ & $\mathrm{FP}\left(^{\circ}\right)$ & $\mathrm{C}(\mathrm{m})$ & $\mathrm{S}(\mathrm{m})$ \\
\hline \multirow{2}{*}{ Short iron } & $\varnothing$ & 36.24 & 42.61 & 1.18 & 3.58 & 2.96 & 3.93 & 113.13 & 6.31 \\
\hline & SD & 0.67 & 1.31 & 0.03 & 1.16 & 1.37 & 1.28 & 4.91 & 2.96 \\
\hline \multirow{2}{*}{ Middle iron } & $\varnothing$ & 37.59 & 47.46 & 1.26 & 4.12 & 3.34 & 3.34 & 131.04 & 8.83 \\
\hline & $\mathrm{SD}$ & 0.31 & 1.66 & 0.04 & 1.08 & 1.55 & 1.86 & 5.96 & 4.88 \\
\hline \multirow{2}{*}{ Long iron } & $\varnothing$ & 39.63 & 52.00 & 1.31 & 4.36 & 3.58 & 2.53 & 153.30 & 11.00 \\
\hline & $\mathrm{SD}$ & 0.28 & 2.43 & 0.06 & 1.49 & 1.37 & 1.57 & 12.60 & 5.69 \\
\hline \multirow{2}{*}{ Driver } & $\varnothing$ & 45.25 & 65.54 & 1.45 & 3.84 & 3.31 & 3.01 & 205.66 & 18.02 \\
\hline & SD & 0.39 & 0.88 & 0.02 & 1.36 & 1.90 & 1.48 & 8.08 & 10.34 \\
\hline
\end{tabular}

CHS - club head speed, IBS - initial ball speed, SF - smash factor, CP - club path, FA - face angle, FP - face to path, $\mathrm{C}$ - resulting ball flight distance - carry, $\mathrm{S}$ - resulting ball flight side deviation from the target line

Table 3. A: The relationship between resulting ball flight distance and the impact factors as determined by Pearson's correlation. B: The relationship between resulting ball flight side deviation from the target line and the impact factors as determined by Pearson's correlation

\begin{tabular}{|c|c|c|c|c|c|}
\hline & \multirow{2}{*}{ Ball flight results } & & \multicolumn{3}{|c|}{ Impact factors } \\
\hline & & & CHS & IBS & SF \\
\hline \multirow{5}{*}{ A } & \multirow{5}{*}{ Resulting ball flight distance } & Short iron & 0.07 & $0.8^{* *}$ & $0.67 *$ \\
\hline & & Middle iron & 0.37 & $0.82 * *$ & $0.81 * *$ \\
\hline & & Long iron & 0.65 & $0.86 * *$ & $0.7^{*}$ \\
\hline & & Driver & 0.64 & $0.87 * *$ & $0.75^{*}$ \\
\hline & & & $\mathrm{CP}$ & FA & $\mathrm{FP}$ \\
\hline \multirow{4}{*}{ B } & \multirow{4}{*}{ Resulting ball flight side deviation } & Short iron & 0.08 & $0.89 * *$ & 0.12 \\
\hline & & Middle iron & -0.1 & $0.67 *$ & 0.04 \\
\hline & & Long iron & 0.2 & 0.59 & 0.05 \\
\hline & & Driver & 0.38 & $0.67^{*}$ & 0.13 \\
\hline
\end{tabular}

CHS - club head speed, IBS - initial ball speed, SF - smash factor, CP - club path, FA - face angle, FP - face to path ${ }^{*} p<0.05,{ }^{* *} p<0.01$

face angle, and the face to path. Significant relationships between the resulting ball flight side deviation and the face angle with each club (short iron: $r=0.89$, $p<0.01$; middle iron: $r=0.67, p=0.05$; driver: $r=0.67$, $p=0.05$ ) were observed. When comparing mutual relationships between the impact factors, a significant relationship was reported between the initial ball speed and the club head speed (driver: $r=0.88, p<0.01$ ). Significant relationships between the initial ball speed and the smash factor for all clubs were also demonstrated (short iron: $r=0.69, p=0.04$; middle iron: $r=0.85$, $p<0.01$; long iron: $r=0.9, p<0.01$; driver: $r=0.66, p=$ 0.05). Significant relationships were found for long iron and driver between the club path and the face angle (long iron: $r=0.79, p=0.01$; driver: $r=0.76, p=0.02$ ) and between the club path and the face to path (long iron: $r=0.71, p=0.03$; driver: $r=0.87, p<0.01$ ).
Table 4 shows the relationships between the impact factors and the players' performance. Significant relationships were found between: handicap and the initial ball speed (short iron: $r=-0.77, p=0.01$; long iron: $r=-0.78, p=0.01$; driver: $r=-0.71, p=0.03$ ), handicap and the face angle (middle iron: $r=0.75, p=$ 0.02), and handicap and the club path (middle iron: $r=0.66, p=0.05)$. A significant relationship was observed for all clubs between the number of strokes and the initial ball speed (short iron: $r=-0.69, p=$ 0.04; middle iron: $r=-0.67, p=0.05$; long iron: $r=$ $-0.74, p=0.02$; driver: $r=-0.71, p=0.03$ ), as well as between the number of strokes and the face angle (middle iron: $r=0.77, p=0.02)$. Significant relationships were demonstrated between the percentage of greens in regulation and the initial ball speed (short iron: $r=$ 0.68, $p=0.04$ ), the smash factor (short iron: $r=0.74$, 
Table 4. The relationship between the impact factors and the players' performance as determined by Pearson's correlation

\begin{tabular}{|c|c|c|c|c|c|c|c|}
\hline \multirow{2}{*}{\multicolumn{2}{|c|}{ Players' performance }} & \multicolumn{6}{|c|}{ Impact factors } \\
\hline & & CHS & IBS & SF & $\mathrm{CP}$ & FA & FP \\
\hline \multirow{4}{*}{ Short iron } & HCP & -0.44 & $-0.77 * *$ & -0.35 & 0.49 & 0.64 & 0.39 \\
\hline & STROKES & -0.35 & $-0.69^{*}$ & -0.3 & -0.01 & 0.5 & 0.22 \\
\hline & GIR & -0.2 & $0.68^{*}$ & $0.74^{*}$ & -0.16 & -0.5 & 0 \\
\hline & FIR & -0.47 & 0.22 & 0.53 & -0.06 & 0.25 & -0.06 \\
\hline \multirow{4}{*}{ Middle iron } & HCP & -0.43 & -0.6 & -0.47 & $0.66^{*}$ & $0.75^{*}$ & 0.14 \\
\hline & STROKES & -0.46 & $-0.67^{*}$ & -0.54 & 0.47 & $0.77^{*}$ & 0.4 \\
\hline & GIR & -0.02 & 0.51 & 0.65 & -0.31 & $-0.72^{*}$ & 0.48 \\
\hline & FIR & -0.49 & 0.12 & 0.49 & -0.41 & -0.25 & 0.33 \\
\hline \multirow{4}{*}{ Long iron } & $\mathrm{HCP}$ & -0.57 & $-0.78 * *$ & -0.64 & 0.32 & 0.53 & 0.47 \\
\hline & STROKES & -0.64 & $-0.74^{*}$ & -0.56 & 0.57 & 0.57 & 0.58 \\
\hline & GIR & 0.02 & 0.62 & $0.75^{*}$ & -0.44 & -0.44 & -0.31 \\
\hline & FIR & -0.35 & 0.33 & 0.61 & -0.43 & -0.12 & -0.47 \\
\hline \multirow{4}{*}{ Driver } & HCP & -0.54 & $-0.71^{*}$ & -0.58 & 0.56 & 0.62 & 0.32 \\
\hline & STROKES & -0.56 & $-0.71^{*}$ & -0.59 & 0.62 & 0.55 & 0.41 \\
\hline & GIR & -0.1 & 0.32 & $0.79 * *$ & $-0.71^{*}$ & -0.49 & $-0.74^{*}$ \\
\hline & FIR & -0.19 & -0.03 & 0.23 & -0.22 & 0.18 & -0.29 \\
\hline
\end{tabular}

CHS - club head speed, IBS - initial ball speed, SF - smash factor, CP - club path, FA - face angle, FP - face to path, HCP - handicap, STROKES - number of strokes, GIR - percentage of greens in regulation, FIR - percentage of fairways in regulation

${ }^{*} p<0.05,{ }^{* *} p<0.01$

Table 5. The relationship between the ball flight results and the players' performance as determined by Pearson's correlation

\begin{tabular}{|c|c|c|c|c|c|c|c|c|}
\hline \multirow{3}{*}{ Players' performance } & \multicolumn{8}{|c|}{ Ball flight results } \\
\hline & \multicolumn{2}{|c|}{ Short iron } & \multicolumn{2}{|c|}{ Middle iron } & \multicolumn{2}{|c|}{ Long iron } & \multicolumn{2}{|c|}{ Driver } \\
\hline & $\mathrm{C}$ & $S$ & $\mathrm{C}$ & $S$ & $\mathrm{C}$ & $S$ & $\mathrm{C}$ & S \\
\hline $\mathrm{HCP}$ & -0.43 & $0.78^{*}$ & -0.48 & 0.23 & $-0.85^{* *}$ & 0.4 & $-0.9 * *$ & 0.57 \\
\hline STROKES & -0.42 & 0.64 & -0.53 & 0.59 & $-0.77^{*}$ & 0.23 & $-0.73^{*}$ & 0.07 \\
\hline GIR & $0.68^{*}$ & -0.37 & 0.57 & -0.44 & 0.56 & -0.44 & 0.64 & -0.52 \\
\hline FIR & 0.16 & 0.25 & 0.24 & 0.27 & 0.03 & 0.08 & 0.31 & -0.23 \\
\hline
\end{tabular}

$\mathrm{C}$ - resulting ball flight distance - carry, $\mathrm{S}$ - resulting ball flight side deviation from the target line, HCP - handicap, STROKES - number of strokes, GIR - percentage of greens in regulation, FIR - percentage of fairways in regulation ${ }^{*} p<0.05,{ }^{*} p<0.01$

$p=0.02$; long iron: $r=0.75, p=0.02$; driver: $r=0.79$, $p=0.01$ ), the club path (driver: $r=-0.71, p=0.03$ ), the face angle (middle iron: $r=-0.72, p=0.03$ ), and the face to path (driver: $r=-0.74, p=0.02$ ). No significant relationship between the percentage of fairways in regulation and any impact factor was revealed.

Table 5 illustrates the relationships between the ball flight results and the players' performance. A significant relationship was found between handicap and the resulting ball flight distance (long iron: $r=-0.85$, $p<0.01$; driver: $r=-0.9, p<0.01$ ), as well as between handicap and the resulting ball flight side deviation from the target line (short iron: $r=0.78, p=0.02$ ). There was a significant relationship between the number of strokes and the resulting ball flight distance (long iron: $r=-0.77, p=0.02$; driver: $r=-0.73, p=0.03$ ) and between the percentage of greens in regulation and the resulting ball flight distance (short iron: $r=$ $0.68, p=0.04$ ). No significant relationship between the percentage of fairways in regulation and the ball flight results was reported. 


\section{Discussion}

The purpose of the present study was to investigate the relationships between players' performance, impact factors, and ball flight results with individual clubs when performing a full swing. The players' performance was tracked during a 1-day tournament after the experiment, in which the participants played 20 strokes with different clubs and were evaluated by the ball flight results and the impact factors. On the basis of the results reported above, with long irons and drivers, players should aim to maximize the distance, and with short irons, they should aim to improve accuracy in order to increase the performance level as defined by handicap. For a longer ball flight distance, amateur players need to increase the quality of contact between the club and the ball, as well as the initial ball speed. For improved accuracy, amateur players need a lower face angle deviation at impact with the ball. Furthermore, the initial ball speed has a strong correlation with performance (handicap, number of strokes per round), although no relationship between handicap and club head speed was found. Because of a significant relationship between the smash factor and performance (the percentage of greens in regulation), the quality of contact with the ball is a priority for hitting the green in regulation. The percentage of fairways in regulation has no relationship with either the ball flight results or impact factors.

The amateur golfers achieved almost identical club head speed with the driver $(45.42 \mathrm{~m} / \mathrm{s})$ in comparison with a study by Sweeney et al. [4], who analysed players of a similar performance level (handicap: 5.7), but lower club head speed than professional golfers $(46.85 \mathrm{~m} / \mathrm{s})$ described in a study by Lewis et al. [24]. Judging by handicap, the participants of this study would be in Betzler and Monk's $2^{\text {nd }}$ performance level category (handicap: 6-12); however, our players also displayed the club head speed that would put them in the $1^{\text {st }}$ performance category (handicap: 0-5) [1]. They achieved higher club head speed with long irons $(39.63 \mathrm{~m} / \mathrm{s}$ vs. $37.64 \mathrm{~m} / \mathrm{s}$ ) than amateur golfers of higher performance (handicap: 0.3) as reported by Bradshaw et al. [19].

\section{Differences in impact factors between clubs}

The impact factors of the face angle, the club path, and the face to path remained unchanged with the varying length of the club, whereas the resulting ball flight side deviation from the target line was increasing (significantly between short iron and driver, between middle iron and driver, and between long iron and driver). The same alignments of the face angle, the club path, and the face to path with different clubs produced larger resulting side deviation, which was especially noticeable between the driver and irons. The increased ball flight distance with the driver likely increased the side deviation setup by the initial conditions of the club-ball interaction. On the basis of these results, we can recommend that amateur golfers play tee shots with irons on short holes with narrow fairways, where accuracy takes precedence.

Relationship between players' performance aspects: handicap, stokes per round, percentage of greens in regulation, and percentage of fairways in regulation

A significant medium-strength relationship was found between handicap and the percentage of greens in regulation $(r=-0.66, p=0.05)$. The more greens in regulation the player hit, the lower their handicap was. Quinn [27] reported a correlation between the percentage of greens in regulation and the average number of strokes per round $(r=-0.62)$ among professional golf players during the season, and Wiseman and Chatterjee [28] demonstrated a correlation between the percentage of greens in regulation and earnings on the PGA Tour in 1990-2004. Because we did not find a relationship between the number of strokes and the percentage of greens in regulation in this study, we suggest that the relation between the number of strokes and the percentage of greens in regulation is a longer-term indicator of performance, which could be observed with amateur golfers over a long term. However, in relation to actual performance (one round score), this relationship depends also on such course conditions as the width of fairways, length of grass in the rough, size and complexity of greens, and wind strength. Furthermore, under these varied (and perhaps more difficult) conditions, short game skills such as chipping and putting are even more important.

\section{Relationship between ball flight results and impact factors}

On the basis of our results, the initial ball speed and the smash factor (quality of contact) during impact are key parameters to produce maximal ball flight distance in amateur golfers. Correct hitting of the ball is necessary for a longer distance. Furthermore, the smash factor has a larger impact than the club head speed on the resulting ball flight distance. However, previous research findings have suggested the club head 
speed as the main determinant $[4,7]$. Players on amateur level and their coaches should focus in practice on the smash factor rather than the club head speed for high ball speed and maximal distance. Our results show that the face angle has the strongest relationship with achieving maximal accuracy of the ball flight. The resulting side deviation of the ball flight is affected mostly by the club path and the face angle during impact [5]. The face angle is the most important factor for the initial launch direction, as shown by other studies (the face angle accounts for approximately $85 \%$ of the initial launch direction) [16]. Coaches should implement drills for keeping the face angle square to target line at impact rather than drills for the club path into their players' training.

When comparing mutual relationships between the impact factors, a significant relationship between the initial ball speed and the smash factor was found for all clubs. Although previous research has identified the club head speed as the main determinant of the initial ball speed [4], a strong relationship between the initial ball speed and the club head speed was observed only for the driver. In the context of our results among amateur golfers, we assume that the initial ball speed with iron clubs is more influenced by the smash factor than the club head speed; on the other hand, ball speed with the driver is more influenced by the club head speed. Another significant relationship was revealed between directional impact factors. The club path correlated strongly with the face angle in long iron and driver and with the face to path again in long iron and driver. The relationship shows that the closer to zero (i.e., more towards the target) the club path is, the closer to zero the face angle or the face to path is. Consequently, the club path depends on the face to path and the face angle depends on the face to path. Players with club paths close to zero also tended to have a similarly low number for face angle. This study does not focus on negative or positive indicators of values, but on the magnitude of these parameters only. However, for description purposes, we report mean values of impact factors which were transferred to absolute values: mean club path -0.43 (outside in), mean face angle -0.76 (closed towards target line), and mean face to path -0.43 (closed towards club path).

Relationship between impact factors and players' performance

A significant medium/strong relationship was found between the initial ball speed parameter and handicap with the short iron, long iron, and driver; and between the initial ball speed parameter and the number of strokes with the short iron, middle iron, long iron, and driver (Tables 4 and 5). On the basis of our results, we suggest that players' performance depends on the initial ball speed produced with any club used. The aim of the player is to achieve a desirable distance with each club; however, individuals who can generate a high enough initial ball speed to achieve the desired distance with shorter clubs are more accurate (e.g., 9 iron instead of 8 iron) because shorter clubs have significantly lower resulting side deviation and therefore players can achieve an improved level of performance [32]. A relationship between handicap and the club head speed, as in a study by Wells et al. [14] (handicap: $2.7 ; r=0.12, p>0.05$ ), was not found. On the other hand, studies reported by Fradkin et al. [7] (handicap: $2-27$; long iron: $r=-0.95, p<0.01$ ), Leary et al. [13] (handicap: 14.5; $r=-0.52, p=0.04$ ), and Williams and Sih [8] (handicap: 0-36; $r=-0.72, p<0.01$ ) demonstrated this relationship significant. We can assume that the relationship between the club head speed and handicap depends on the performance level of players: high handicaps, low handicaps, or elite players.

A significant medium/strong relationship between the percentage of greens in regulation and the smash factor with short iron, long iron, and driver was found. On this basis, we suggest that the quality of contact with the ball is a key impact factor for hitting the green in regulation.

A significant medium relationship was observed between the percentage of greens in regulation and the face angle with the middle iron, between the percentage of greens in regulation and the club path with the driver, and between the percentage of greens in regulation and the face to path with the driver. Although this study showed only a strong relationship between the resulting ball flight side deviation and face angle, other studies have presented the importance of the club path [5]. This relationship has been demonstrated to some extent here, where both face angle and club path with the driver exhibited a medium-strength relationship with the percentage of greens in regulation.

\section{Relationship between ball flight results and players' performance}

A strong and significant relationship between the resulting ball flight distance and the players' performance as defined by handicap and the number of strokes was found with the long iron and driver, as in studies by Sell et al. [33] (with the driver: $r=-0.48$, $p<0.01)$ and by Wiren [34] $(r=-0.61)$. Conversely, with 
the short iron, a strong relationship was observed between handicap and the resulting ball flight side deviation from the target line. This relationship indicates that with long irons and drivers, players should focus on increasing the total distance, and with short irons, they should focus on becoming more accurate to increase performance as defined by handicap. This is new knowledge that allows coaches to use a different training strategy for each type of golf club.

A significant medium correlation between the percentage of greens in regulation and the resulting ball flight distance was revealed with short irons. The greater total distance a player produced with short irons, the more greens in regulation they hit. This relationship is in contrast to what we found when defining performance by handicap, where the resulting ball flight distance with longer clubs and the ball flight side deviation with short irons correlated with handicap. In relation to the performance as defined by the percentage of greens in regulation, the resulting ball flight distance is more important with short irons.

\section{Limitations}

Whilst the results presented above add to our understanding of key golf performance parameters, the study is not without its limitations. Players' performance could be tracked longitudinally, which would allow to assess more than one tournament across a variety of course and weather conditions. Another limitation may be the lack of generalizability of the results to other demographic groups, e.g. women golfers or elite/professional golfers. From the perspective of the experiment, the participants played from a practice mat in the swing test. This potentially lacks ecological validity as hitting from a mat is different to playing from grass. Future research should be conducted on a grass surface.

\section{Conclusions}

On the basis of our results, we conclude that the players' performance depends on maximal ball flight distance with drivers and long irons, and on accuracy with short irons. Ball flight distance depends on the quality of contact between the club and the ball (the smash factor) and the initial ball speed. Accuracy depends mostly on the angle between the target line and leading edge of the club face (face angle). Coaches should focus on the smash factor (quality of contact with the ball), e.g. by choosing drills to correct mistakes in golf swing that lead to a low quality of contact with the ball, instead of drills to speed up the club head. In practice, amateur golfers and their coaches should concentrate more on controlling the face angle at impact rather than club path for improved accuracy. In order to extend our understanding across performance levels, future research should concern the relationship between the variability of impact factors and the resulting ball flight results among amateur and elite amateur golf players.

\section{Acknowledgments}

We would like to thank all participants of the study.

\section{Funding}

The study was supported by Charles University, project GA UK No. 1466120; by the grant SVV No. 260599; and by research grant UNCE/HUM/032.

\section{Disclosure statement}

No author has any financial interest or received any financial benefit from this research.

\section{Conflict of interest}

The authors state no conflict of interest.

\section{References}

1. Betzler NF, Monk SA, Wallace ES, Otto SR. Variability in clubhead presentation characteristics and ball impact location for golfers' drives. J Sports Sci. 2012;30(5): 439-448; doi: 10.1080/02640414.2011.653981.

2. Horan SA, Evans K, Kavanagh JJ. Movement variability in the golf swing of male and female skilled golfers. Med Sci Sports Exerc. 2011;43(8):1474-1483; doi: 10.1249/ MSS.0b013e318210fe03.

3. Langdown BL, Bridge M, Li F-X. Movement variability in the golf swing. Sports Biomech. 2012;11(2):273-287; doi: 10.1080/14763141.2011.650187.

4. Sweeney M, Mills P, Alderson J, Elliott B. The influence of club-head kinematics on early ball flight characteristics in the golf drive. Sports Biomech. 2013;12(3):247258; doi: 10.1080/14763141.2013.772225.

5. TrackMan. TrackMan University. 2019. Available from: https://trackmanuniversity.com.

6. Baugher CD, Day JP, Burford EW Jr. Drive for show and putt for dough? Not anymore. J Sports Econ. 2016; 17(2):207-215; doi: 10.1177/1527002514528517.

7. Fradkin AJ, Sherman CA, Finch CF. How well does club head speed correlate with golf handicaps? J Sci Med Sport. 2004;7(4):465-472; doi: 10.1016/S1440-2440(04) 80265-2.

8. Williams KR, Sih BL. Changes in golf clubface orientation following impact with the ball. Sports Eng. 2002; 5(2):65-80; doi: 10.1046/j.1460-2687.2002.00093.x.

9. Alvarez M, Sedano S, Cuadrado G, Redondo JC. Effects of an 18-week strength training program on low- 
handicap golfers' performance. J Strength Cond Res. 2012;26(4):1110-1121; doi: 10.1519/JSC.0b013e3182 2 dfa7d.

10. Fletcher IM, Hartwell M. Effect of an 8-week combined weights and plyometrics training program on golf drive performance. J Strength Cond Res. 2004;18(1):59-62; doi: 10.1519/1533-4287(2004)018<0059:eoawcw $>2$. 0. co;2.

11. Hetu FE, Christie CA, Faigenbaum AD. Effects of conditioning on physical fitness and club head speed in mature golfers. Percept Mot Skills. 1998;86(3 Pt 1):811815; doi: 10.2466/pms.1998.86.3.811.

12. Joyce C. The most important "factor" in producing clubhead speed in golf. Hum Mov Sci. 2017;55:138-144; doi: 10.1016/j.humov.2017.08.007.

13. Leary BK, Statler J, Hopkins B, Fitzwater R, Kesling T, Lyon J, et al. The relationship between isometric forcetime curve characteristics and club head speed in recreational golfers. J Strength Cond Res. 2012;26(10): 2685-2697; doi: 10.1519/JSC.0b013e31826791bf.

14. Wells JET, Mitchell ACS, Charalambous LH, Fletcher IM. Relationships between highly skilled golfers' clubhead velocity and force producing capabilities during vertical jumps and an isometric mid-thigh pull. J Sports Sci. 2018;36(16):1847-1851; doi: 10.1080/026 40414.2018.1423611.

15. Doan BK, Newton RU, Kwon Y-H, Kraemer WJ. Effects of physical conditioning on intercollegiate golfer performance. J Strength Cond Res. 2006;20(1):62-72; doi: 10.1519/R-17725.1.

16. Tucker CB, Anderson R, Kenny IC. Is outcome related to movement variability in golf? Sports Biomech. 2013; 12(4):343-354; doi: 10.1080/14763141.2013.784350.

17. Pelz D. Dave Pelz's short game bible: master the finesse swing and lower your score. London: Aurum Press Limited; 1999.

18. Miura K. Mapping clubhead to ball impact and estimating trajectory. In: Thain E (ed.), Science and golf IV: Proceedings of the World Scientific Congress of Golf. Abingdon: Routledge; 2002; 490-500.

19. Bradshaw EJ, Keogh JWL, Hume PA, Maulder PS, Nortje J, Marnewick M. The effect of biological movement variability on the performance of the golf swing in highand low-handicapped players. Res Q Exerc Sport. 2009; 80(2):185-196; doi: 10.1080/02701367.2009.10599552.

20. Fedorcik GG, Queen RM, Abbey AN, Moorman CT $3^{\text {rd }}$, Ruch DS. Differences in wrist mechanics during the golf swing based on golf handicap. J Sci Med Sport. 2012; 15(3):250-254; doi: 10.1016/j.jsams.2011.10.006.

21. Robertson S, Gupta S, Kremer P, Burnett AF. Development and measurement properties of a putting skill test for high-level golf. Eur J Sport Sci. 2015;15(2):125133; doi: 10.1080/17461391.2014.932014.

22. Keogh JWL, Hume PA. Evidence for biomechanics and motor learning research improving golf performance. Sports Biomech. 2012;11(2):288-309; doi: 10.1080/14 763141.2012 .671354 .
23. Alexander DL, Kern W. Drive for show and putt for dough? An analysis of the earnings of PGA Tour golfers. J Sports Econ. 2005;6(1):46-60; doi: 10.1177/152700 2503260797.

24. Lewis AL, Ward N, Bishop C, Maloney S, Turner AN. Determinants of club head speed in PGA professional golfers. J Strength Cond Res. 2016;30(8):2266-2270; doi: 10.1519/jsc.0000000000001362.

25. Moy RL, Liaw T. Determinants of professional golf tournament earnings. Am Econ. 1998;42(1):65-70; doi: $10.1177 / 056943459804200106$.

26. James N. The statistical analysis of golf performance. Int J Sports Sci Coach. 2007;2(1 Suppl.):231-249; doi: 10.1260/174795407789705424.

27. Quinn RJ. Exploring correlation coefficients with golf statistics. Teach Stat. 2006;28(1):10-13; doi: 10.1111/ j.1467-9639.2006.00229.x.

28. Wiseman F, Chatterjee S. Comprehensive analysis of golf performance on the PGA Tour: 1990-2004. Percept Mot Skills. 2006;102(1):109-117; doi: 10.2466/pms. 102.1.109-117.

29. Harriss DJ, Atkinson G. Ethical standards in sport and exercise science research: 2016 update. Int J Sports Med. 2015;36(14):1121-1124; doi: 10.1055/s-0035-15 65186.

30. Leach RJ, Forrester SE, Mears AC, Roberts JR. How valid and accurate are measurements of golf impact parameters obtained using commercially available radar and stereoscopic optical launch monitors? Measurement. 2017;112:125-136; doi: 10.1016/j.measurement.2017. 08.009 .

31. Portney LG, Watkins MP. Foundations of clinical research: applications to practice. Upper Saddle River: Pearson/Prentice Hall; 2009.

32. James N, Rees GD. Approach shot accuracy as a performance indicator for US PGA Tour golf professionals. Int J Sports Sci Coach. 2008;3(1 Suppl.):145-160; doi: 10.1260/174795408785024225.

33. Sell TC, Tsai Y-S, Smoliga JM, Myers JB, Lephart SM. Strength, flexibility, and balance characteristics of highly proficient golfers. J Strength Cond Res. 2007;21(4): 1166-1171; doi: 10.1519/R-21826.1.

34. Wiren G. Human factors influence the golf drive for distance. Doctoral thesis. Eugene: University of Oregon; 1968. 